\title{
Positive association to IgE levels and a physical map of the $13 q 14$ atopy locus
}

\author{
Gavin G Anderson ${ }^{1,2}$, Nicholas I Leaves ${ }^{1,2}$, Sumit Bhattacharyya ${ }^{1}$, Youming Zhang $^{1}$, \\ Valerie Walshe ${ }^{1}$, John Broxholme ${ }^{1}$, Gonçalo Abecasis ${ }^{1}$, Elaine Levy ${ }^{1}$, Michael Zimmer ${ }^{1}$, \\ Roger $\operatorname{Cox}^{1}$ and William OCM Cookson ${ }^{\star}, 1$
}

\footnotetext{
${ }^{1}$ The Welcome Trust Centre for Human Genetics, University of Oxford, Roosevelt Drive, Headington, Oxford, OX3 7BN, UK
}

Linkage of atopy and associated traits to a locus on chromosome 13q14 has been identified by several studies in diverse populations. We have previously shown the putative atopy gene to be contained within an interval of approximately $5 \mathrm{Mb}$ flanked by $D 135328$ and D13S1269 and centred on D13S273. We have now extended this work using a top-down approach to physical mapping. A YAC contig was constructed covering the D13S328 and D13S1269 interval. Thirty-one ESTs were mapped to the contig. We constructed a BAC and PAC contig flanking $D 13 S 273$ by $\sim 750 \mathrm{~kb}$ in either direction. The interval contained 27 of the 31 ESTS from the YAC contig. Seven previously unknown microsatellites were recovered and then typed in two subject panels. A positive association between the total serum Immunoglobulin E concentration and the novel USAT24G1 microsatellite was discovered $\left(P_{\text {corrected }}<0.005\right)$ and replicated in a second panel of families. The discovery of a region of positive association within the BAC/PAC contig will permit identification of the atopy gene from this locus.

European Journal of Human Genetics (2002) 10, 266-270. DOI: 10.1038/sj/ejhg/5200801

Keywords: atopy; IgE; chromosome 13; physical map; TDT

\section{Introduction}

Atopic asthma is the most common chronic disease of childhood in the UK. ${ }^{1}$ Atopy and asthma result from an interaction between environmental and genetic factors and show a complex mode of inheritance. Segregation analyses and twin studies indicate that asthma and atopy have a significant genetic component. ${ }^{2,3}$ These diseases are likely to be influenced by genes of moderate effect in addition to polygenes. Several regions of genetic linkage have been recognised by different groups, and a number of definite associations have been made to candidate genes. ${ }^{2}$

*Correspondence: Professor William Cookson, Wellcome Trust Centre for Human Genetics, Roosevelt Drive, Oxford OX3 7BN, UK.

Tel: +44 1865 287607; Fax: +44 1865 287578;

E-mail: wocc@gwmail.jr2.ox.ac.uk

${ }^{2}$ These authors contributed equally to the work

Received 21 September 2001; revised 18 January 2002; accepted 1 March 2002
We have previously identified a linkage region on chromosome 13q14 in a genome screen for asthma and atopy and their associated traits in two populations. ${ }^{4}$ The maximum linkage to atopy was identified at the microsatellite marker D13S153. No obvious candidate gene is known to exist within this region. A report of genetic linkage to the total serum Immunoglobulin E concentration (IgE) at the nearby Esterase D locus pre-existed this work, ${ }^{5}$ and linkage has also been reported in other families with asthma and allergy. ${ }^{6,7}$ Linkage to $13 q 14$ has also been observed to house dust mite allergy in children with asthma ${ }^{8}$ and to children with atopic dermatitis. ${ }^{9}$ Kimura et $\mathrm{al}^{7}$ showed linkage in Japanese families to D13S153, and identified a possible positive transmission association to atopic asthma at this marker, suggesting the proximity of a gene influencing these phenotypes.

We have subsequently created a high-density saturation genetic map of 7.5-cM of the 13q14 locus, and have shown localisation of the gene to be bordered by D13S328 and D13S1269. ${ }^{10}$ 
Cytogenetic abnormalities at 13q14 have been noted in around $25 \%$ of cases of B-cell chronic lymphocytic leukaemia (B-CLL). ${ }^{11}$ The most commonly deleted region extends $1 \mathrm{Mb}$ between the markers D13S272 and D13S25, ${ }^{12,13}$ although the locus has been placed more in a more centromeric position between D13S273 and D13S272 by other authors. ${ }^{14}$ The BCLL locus therefore extends into our region of interest, and we have integrated physical maps of this locus into our results.

\section{Materials and methods}

\section{Families and phenotype}

Two panels of subjects, AUS1 and UK1, were used in the genetic analysis of the locus. Their recruitment and phenotypic characteristics have previously been described. ${ }^{4}$ Our genome screen was based on the AUS1 Panel (the primary data set) comprising 80 nuclear families containing 364 subjects with 172 sib-pairs. ${ }^{4}$ The UK1 Panel (the replication set) contained 77 nuclear and seven extended pedigrees comprising 380 subjects with 268 sib-pairs. ${ }^{15}$

The total serum immunoglobulin E concentration was $\log _{\mathrm{e}}$ transformed and analysed as a quantitative trait $\left(\log _{\mathrm{e}} \operatorname{IgE}\right)$.

\section{Libraries and library screening}

The yeast artificial chromosomes (YACs) used were from the CEPH mega YAC library (www.cephb.fr/services). YACs were identified by electronic screening for STSs (http://carbon.wi.mit.edu.8000/cgi-bin/contig/phys_map) and by PCR screening as described. ${ }^{16,17}$ The human Bacterial Artificial Chromosome (BAC) and P1-derived Artificial Chromosome (PAC) libraries were the CITB BAC Pools Release IV library (www.resgen.com/products/CITBBAC_pf.php3) and the Pieter DeJong Human PAC library (www.resgen.com/products/ RPCIHum.php3). PCR screening and subculturing of the libraries was performed as described by the distributor (Research Genetics Inc., Huntsville, AL, USA). All YACs, BACs and PACs used in this project were subcultured to purity, checked for the presence of correct insert by PCR and then stored in LB broth with $30 \%$ glycerol at $-80^{\circ} \mathrm{C}$.

\section{Construction of contigs}

YACs were sized by pulsed field gel electrophoresis (PFGE) and Southern blotting, and were assayed for the presence of NotI and SalI restriction sites. A preliminary single-linked contig was generated between markers D13S328 and D13S1269 by selecting YAC clones for known microsatellites (D13S161, D13S153, D13S1307, D13S165, D13S273, D13S272, D13S1269, D13S284, D13S1325 and D13S1305). A higher density of PCR markers including further microsatellites, STSs and ESTs were then added using the YACs as DNA template. ESTs and STSs putatively located within the region were derived from a genome-wide radiation hybrid map (www.ncbi.nlm.nih.gov/genemap).

The BAC and PAC contig was centred on the marker D13S273, which was close to the peak of linkage. ${ }^{10}$ The YAC framework map indicated that 20 PCR markers (microsatellites, ESTs and STSs) were present within this region. These makers were used to screen the BAC and PAC libraries. Gaps were closed by IRE bubble $\mathrm{PCR}^{18,19}$ to recover BAC/PAC clone ends then designing new STSs and re-screening the libraries. NotI and SalI restriction sites were used to align the BAC and PAC clones with one another and also with the YAC map. We aimed to achieve a greater than 2-fold depth to the entire contig. PACs, ESTs and STSs from the B-CLL locus contig ${ }^{20,21}$ were integrated into the right hand side (qtel) of our contig.

\section{Microsatellite identification and genotyping}

IRE bubble linkers were employed to rescue novel microsatellites. ${ }^{19}$ Repeats were obtained by PCR using the bubble primer and the following dinucleotide primers: $(\mathrm{CA})_{11} \mathrm{~A}$, $(\mathrm{CA})_{11} \mathrm{~T},(\mathrm{AC})_{11} \mathrm{~T},(\mathrm{CA})_{11} \mathrm{G},(\mathrm{AC})_{11} \mathrm{G},(\mathrm{AG})_{11} \mathrm{~A},(\mathrm{AG})_{11} \mathrm{~T}$, $(A G)_{11} G$, $(A G)_{11} C$; trinuceotide primers: $(G G C)_{8}$, $(A A C)_{8}$, $(\mathrm{AGC})_{10}$ and tetranucleotide primers: (AAAB) $)_{10}$, (AATG) $)_{5}$, $(\mathrm{AGAT})_{5},(\mathrm{AAAG})_{5},(\mathrm{ATCC})_{5},(\mathrm{AAGG})_{5}$ and $(\mathrm{ACAT})_{5}$. Discrete PCR products were sequenced and the length of microsatellite repeat was assessed.

Informative microsatellites were typed in both study panels A and B. Genotyping was carried out using a high throughput fluorescent PCR based method. ${ }^{22}$ Primers are available on http://www.well.ox.ac.uk/asthma/.

\section{Statistical Analyses}

Mendelian inheritance was checked using Genetic Analysis Software version 2.1 (GAS) ${ }^{23}$ (http://www.linkage.rockefeller. edu/soft/list2.html\#g) and discrepancies were re-examined and resolved using GENOTYPER ${ }^{(i m}$. Statistical analyses of association to the total serum IgE were carried out by the QTDT program, which allowed variance-components testing of family-based samples for association and transmission disequilibrium as described by Abecasis et $a l^{24}$ Association was tested in all family members (Total Association Model $^{24}$ ) to maximise use of the available data. To minimise multiple comparisons, multi-allelic tests of association were performed. ${ }^{24} P$ values in the primary dataset were corrected by a Bonferroni multiplication with the number of markers tested $(n=22)$.

\section{Results}

YAC contig construction

A YAC clone contig was constructed across the region spanning D13S328 and D13S1269. Thirteen YAC clones (903a12, 847g8, 830c8, 851f1, 775c8, 868f11, 959a12, $745 \mathrm{e} 3,747 \mathrm{~b} 8,857 \mathrm{c} 5,895 \mathrm{~b} 11,879 \mathrm{f5}$ and 935g2) were identified by electronic and PCR screening. The contig extended for approximately $6 \mathrm{Mb}$ between $D 135328$ and SHGC-13595. The approximate size of the region between the extreme microsatellites (D13S328 and D13S1269) was $4 \mathrm{Mb}$. FISH showed two clones $(775 \mathrm{c} 8$ and $745 \mathrm{e} 3)$ to be chimeric: the remaining clones were used to draw a non-chimeric, 
multiply linked, minimum tiling path between $D 13 S 328$ and SHGC-13595. The contig contained 21 polymorphic markers, five anonymous STSs and 31 ESTs arranged into 12 bins: WI6333, WI-9598 and D13S272 could not be binned. The microsatellite order was estimated using a combination of contig STS content and MULTIMAP to be: D13S328 D13S168 - D13S287 - D13S161 - D13S153 - D13S164 D13S1307 - D13S165 - D13S273 - D13S272 - D13S1269 D13S262 - D13S284 - D13S788 - D13S268 - D13S1325 ATA17CO6 - D13S1305.

\section{BAC and PAC contig construction}

Chromosome walking produced a complete contig for the region $D 13 S 1307$ to $D 13 S 272$ (Figure 1). The contig consisted of 28 BACs and 8 PACs. There were three regions with only a single clone spanning a gap which could not be deepened despite repeated library screening. FISH determined that 5 of 41 clones were not present on 13q14. These clones were discarded. NotI and SalI restriction digest data was completed for 34 clones (Figure 1). The average insert size of the BACs and PACs was $117.9 \mathrm{~kb}$ and $117.4 \mathrm{~kb}$ respectively. We identified five NotI sites and seven SalI sites. They clustered in two locations, one near the junction of clones HPAC35C13, HBAC339L13 and HBAC401J1, and the other just centromeric to HBAC352L21. Summing the restriction fragments together with non-overlapping clones gave an estimate of the contig size to be $\sim 1.5 \mathrm{Mb}$. In addition, the physical distances separating the four pre-existing microsatellites could be estimated to be: D13\$1307 - $600 \mathrm{~kb}$; D13S165 - $600 \mathrm{~kb}$; D13S273 - $300 \mathrm{~kb}$; D13S272. Twentyseven of the 31 ESTs placed on the YAC map were proven to be on the PAC and BAC contig (Figure 1).

\section{Microsatellite recovery}

Twenty-five novel microsatellites were recovered, seven of which were polymorphic when typed in 32 unrelated healthy volunteers. These seven new markers had mean heterozygosities between 0.601 and 0.748 .

\section{Family panel characteristics}

The mean age of the children in the primary (AUS1) panel was 12.6 years $( \pm$ SE 1.3$)$, their geometric mean IgE was $55.7 \pm 1.1 \mathrm{IU} / \mathrm{l}: 12 \%$ were asthmatic. The mean age of the AUS1 parents was $39.9 \pm 0.36$ years, their geometric mean IgE was $24.8 \pm 1.1 \mathrm{IU} / \mathrm{l}: 14 \%$ were asthmatic. The mean age of the children in the replication panel (UK1) was $16.8 \pm 10.9$ years and their geometric mean IgE was $115.2 \pm 1.1 \mathrm{IU} / \mathrm{l}: 56 \%$ were asthmatic. The mean age of the UK1 parents was $39.6 \pm 0.70$ years, their geometric mean IgE was $56.6 \pm 1.1 \mathrm{IU} / \mathrm{l}$ : $47 \%$ were asthmatic.

\section{Genetic analyses}

The seven novel microsatellites were typed together with 15 microsatellites already in the public domain (all that were

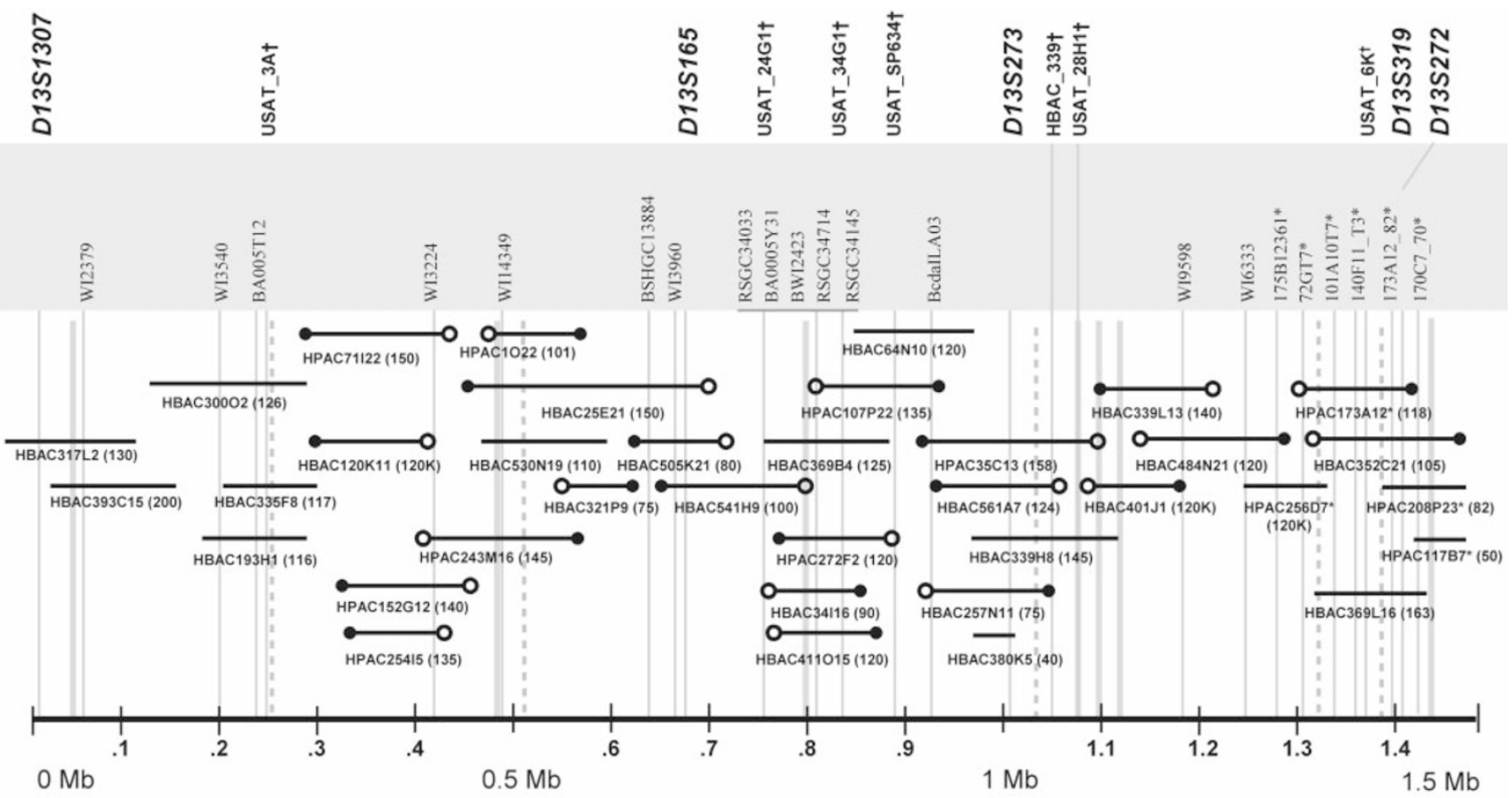

Figure $113 q 14$ atopy locus BAC/PAC contig. The Notl restriction sites are delineated by thick dashed lines and Sall by thick full lines. The size $(\mathrm{kb})$ of the BAC/PAC is given in parenthesis. SP6 BAC/PAC ends are represented by the open circles, T7 by filled circles. ESTs, STSs and PACs derived from the CLL locus are denoted by * (Kalachikov et $a l^{21}$ ). Novel microsatellites are denoted by $\dagger$. 
available at the time of typing) (Table 1). We tested these markers for evidence of association to the quantitative trait of total serum IgE concentration (Table 1). A highly significant association was detected to the $\log _{\mathrm{e}} \operatorname{IgE}$ at USAT24G1, within $200 \mathrm{~kb}$ of D13S273. This figure remained significant when corrected for multiple testing of 22 markers $\left(P_{\text {corrected }}\right.$ $=0.0044)$. Parent of origin effects were not detected. Adjoining microsatellites in the USAT24G1 - D13S273 interval showed weaker association. The associations were replicated in the second panel of families.

\section{Discussion}

The results of our study build upon the previously demonstrated linkage of atopy and related phenotypes to chromosome $13 q 144^{4,6,7,25}$ The finding of a positive association between the total serum IgE concentration and microsatellites in a limited region of $200 \mathrm{~kb}$ represents a further step in fine localisation of the disease from the $5 \mathrm{~cm}, 1 \mathrm{LOD}$ score support unit identified by Bhattacharyya et al. ${ }^{10}$

The construction of the PAC/BAC map was assisted by the work of various groups investigating the B-CLL locus. ${ }^{14,20,21,26}$ Kalachikov et al $^{21}$ constructed a 13 clone PAC contig and a cosmid contig across the minimally deleted region in B-CLL at 13q14.3. This PAC contig spanned $560 \mathrm{~kb}$

Table 1 Summary of association testing between the total serum IgE concentration and microsatellite markers across the YAC contig covering the chromosome $13 q 14$ atopy locus

\begin{tabular}{|c|c|c|c|c|}
\hline \multirow[t]{2}{*}{ Marker } & \multirow[t]{2}{*}{$\begin{array}{c}\text { Heterozygosity } \\
\text { (\%) }\end{array}$} & \multirow[t]{2}{*}{$\begin{array}{l}\text { Location } \\
\text { (Mbp) }\end{array}$} & \multicolumn{2}{|c|}{$\begin{array}{l}\text { Association to } \lg E \\
\text { (multi-allelic test) }\end{array}$} \\
\hline & & & AUS1 & UK1 \\
\hline D13S168 & 81.2 & 0 & - & \\
\hline D13S161 & 64.3 & 0.20 & - & \\
\hline D13S287 & 66.2 & 0.30 & - & \\
\hline D13S153 & 89.4 & 0.80 & - & \\
\hline D1351307 & 69.6 & 1.35 & - & \\
\hline USATEA & 43.0 & 1.40 & - & \\
\hline D13S165 & 81.6 & 1.45 & - & \\
\hline USAT24G 1 & 61.7 & 1.50 & 0.0002 & 0.028 \\
\hline USAT34G 1 & 49.4 & 1.60 & 0.082 & - \\
\hline S34 & 36.1 & 1.65 & - & 0.095 \\
\hline D13S273 & 75.5 & 1.70 & 0.094 & 0.023 \\
\hline USAT28H1 & 74.7 & 1.75 & - & \\
\hline USATHBAC 339 & 65.0 & 1.80 & - & \\
\hline MGG & 70.6 & 1.90 & - & \\
\hline USAT6K & 91.2 & 1.95 & - & \\
\hline D13S272 & 70.5 & 2.05 & - & \\
\hline D1351269 & 70.3 & 2.75 & - & \\
\hline D13S262 & 80.5 & 2.85 & - & \\
\hline D13S284 & 88.0 & 3.35 & - & \\
\hline D13S1325 & 81.0 & 3.95 & - & \\
\hline D13ATA17 & 60.0 & 4.55 & - & \\
\hline D1351305 & 74.0 & 5.15 & - & \\
\hline
\end{tabular}

Novel microsatellites recovered from the contig are shown in bold. Non-significant results $(P>0.1)$ are shown as -. Markers were tested in the primary (AUS1) panel of families, and those showing positive results were tested in the secondary (UK1) panel between markers D13S1150 and D13S25 and is proximally overlapping with our atopy locus by approximately $200 \mathrm{~kb}$ at the distal (telomeric) end of our contig (around marker D13S272). Clones from the Kalachikov contig were validated and then incorporated into the distal $200 \mathrm{~kb}$ of our contig.

A NotI and SalI restriction map was created to help orientate and estimate the overlap and total size of the clones in the contig and to identify potential CpG islands at the $5^{\prime}$ end of genes. ${ }^{27,28}$ Five NotI and seven SalI sites were identified and appeared to cluster in two regions, one immediately centromeric to D13S272 (in the critical deletion of the B-CLL locus) and the second $50-150 \mathrm{~kb}$ telomeric to D13S273. A number of putative transcripts are close by the first cluster of CpG islands but the second cluster is only close to the EST WI9598. This may indicate that there are further transcripts to be identified from the region.

The distribution of ESTs mapped to the locus was irregular, perhaps reflecting variations in gene density or the detection of multiple fragments of genes. A cluster of ESTs was observed around BAC 346I16 and a second cluster around marker D13S272. The latter cluster is likely to be at least in part due to the large transcript mapping effort made by the groups investigating the B-CLL locus. It is likely that not all 27 ESTs represent unique genes, and may represent multiple fragments or splice variants of the same transcripts, contaminating genomic sequence or pseudogenes.

Seven novel microsatellites were recovered from the final BAC/PAC map and genotyped together with public domain microsatellites. The most significant association to the serum IgE in the primary dataset was to the novel microsatellite USAT24G1. The association to this marker remained highly significant even after correcting for the number of markers tested, and was also seen in the replication panel of families.

The extent and distribution of LD is not constant over the genome, but 'useful' LD (that is able to detect association with disease) extends for an average of approximately $50 \mathrm{~kb}$ in this region. ${ }^{29}$ The limit of detection of linkage disequilibrium (LD) between a disease and a marker given our sample size is likely to be less than $100 \mathrm{~kb},{ }^{29,30}$ suggesting that an atopy gene is within $100 \mathrm{~kb}$ in either direction of USAT24l.

The statistical significance of the weak associations of IgE levels to other microsatellites in the $200 \mathrm{~kb}$ USAT24G1 D13S273 interval is uncertain, but may be taken as an indication that this entire interval should be investigated with a dense SNP map, and for the comprehensive characterisation of all transcripts. The chromosome 13q14 region encompassing our locus is being currently sequenced at the Sanger Centre, Cambridge (http://www.sanger.ac.uk/ HGP/Chr13/). The results are in draft form at present.

\author{
Acknowledgments \\ This work was funded by the Wellcome Trust
}




\section{References}

1 Strachan DP, Anderson HR, Limb ES, O'Neill A, Wells, N: A national survey of asthma prevalence, severity, and treatment in Great Britain. Arch Dis Child 1994; 70: 174-178.

2 Cookson WO, Moffatt MF: Genetics of asthma and allergic disease. Hum Mol Genet 2000; 9: 2359-2364.

3 Sandford A, Weir T, Pare P: The genetics of asthma. Am J Respir Crit Care Med 1996; 153: 1749-1765.

4 Daniels SE, Bhattacharrya S, James A, et al: A genome-wide search for quantitative trait loci underlying asthma. Nature 1996; 383: $247-250$.

5 Eiberg H, Lind P, Mohr J, Nielsen LS: Linkage relationship between the human immunoglobulin $\mathrm{E}$ polymorphism and marker systems. Cytogenet Cell Genet 1985; 40: 622.

6 Ober C, Cox NJ, Abney M, et al: Genome-wide search for asthma susceptibility loci in a founder population. The Collaborative Study on the Genetics of Asthma. Hum Mol Genet 1998; 7: 1393 1398.

7 Kimura K, Noguchi E, Shibasaki M, et al: Linkage and association of atopic asthma to markers on chromosome 13 in the Japanese population. Hum Mol Genet 1999; 8: 1487-1490.

8 Hizawa N, Friedhoff L, Chiu Y, et al: Genetic regulation of Dermatophagoides pteronyssinus-specific IgE responsiveness: a genome-wide multipoint linkage analysis in families recruited through 2 asthmatic sibs. Collaborative Study on the Genetics of Asthma (CSGA). J Allergy Clin Immunol 1998; 102: 436-442.

9 Beyer KWU, Freidhoff L, Nickel R, et al: Evidence for linkage of chromosome 5q31-q33 and 13q12-q14 markers to atopic dermatitis. J Allergy Clin Immunol 1998; 101: 152.

10 Bhattacharyya S, Leaves NI, Wiltshire S, Cox R, Cookson WO: A high-density genetic map of the chromosome 13q14 atopy locus. Genomics 2000; 70: 286-291.

11 Juliusson G, Gahrton G: Chromosome aberrations in B-cell chronic lymphocytic leukemia. Pathogenetic and clinical implications. Cancer Genet Cytogenet 1990; 45: 143-160.

12 Liu Y, Hermanson M, Grander D, et al: 13q deletions in lymphoid malignancies. Blood 1995; 86: 1911-1915.

13 Stilgenbauer S, Leupolt E, Ohl S, et al: Heterogeneity of deletions involving RB-1 and the D13S25 locus in B-cell chronic lymphocytic leukemia revealed by fluorescence in situ hybridization. Cancer Res 1995; 55: 3475 - 3477.

14 Corcoran MM, Rasool O, Liu Y, et al: Detailed molecular delineation of 13q14.3 loss in B-cell chronic lymphocytic leukemia. Blood 1998; 91: 1382-1390.

15 Hill MR, James AL, Faux JA, et al: Fc epsilon RI-beta polymorphism and risk of atopy in a general population sample. Bmj 1995; 311: $776-779$.
16 Fischer SG, Cayanis E, Russo JJ, et al: Assembly of ordered contigs of cosmids selected with YACs of human chromosome 13. Genomics 1994; 21: 525-537.

17 Fischer SG, Cayanis E, de Fatima Bonaldo M, et al: A highresolution annotated physical map of the human chromosome 13q12-13 region containing the breast cancer susceptibility locus BRCA2. Proc Natl Acad Sci USA 1996; 93: 690-694.

18 Munroe DJ, Haas M, Bric E, et al: IRE-bubble PCR: a rapid method for efficient and representative amplification of human genomic DNA sequences from complex sources. Genomics 1994; 19: $506-514$.

19 Merriman T, Twells R, Merriman M, et al: Evidence by allelic association-dependent methods for a type 1 diabetes polygene (IDDM6) on chromosome 18q21. Hum Mol Genet 1997; 6: 1003 1010.

20 Bezieau S, Devilder MC, Rondeau G, et al: Assignment of 48 ESTs to chromosome 13 band q14.3 and expression pattern for ESTs located in the core region deleted in B-CLL. Genomics 1998; 52 : $369-373$.

21 Kalachikov S, Migliazza A, Cayanis E, et al: Cloning and gene mapping of the chromosome 13q14 region deleted in chronic lymphocytic leukemia. Genomics 1997; 42: 369-377.

22 Reed PW, Davies JL, Copeman JB, et al: Chromosome-specific microsatellite sets for fluorescence-based, semi-automated genome mapping. Nat Genet 1994; 7: 390-395.

23 Young A: Genetic Analysis Systems. in Genetic Analysis Systems, Version 2.1. Oxford University, 1996.

24 Abecasis GR, Cardon LR, Cookson WO: A general test of association for quantitative traits in nuclear families. $A m$ Hum Genet 2000; 66: 279-292.

25 A genome-wide search for asthma susceptibility loci in ethnically diverse populations. The Collaborative Study on the Genetics of Asthma (CSGA). Nat Genet 1997; 15: 389-392.

26 Hawthorn LA, Cowell JK: Construction of a bacterial artificial chromosome (BAC) contig across the minimally deleted region in 13q14.3 in B-cell chronic lymphocytic leukemia. Ann Hum Genet 1998; 62 ( Pt 5): 401-409.

27 Bird AP: CpG islands as gene markers in vertebrate nucleus. Trends Genet 1987; 3: 342-347.

28 Larsen F, Gundersen G, Lopez R, Prydz H: CpG islands as gene markers in the human genome. Genomics 1992; 13: 1095 - 1107.

29 Abecasis GR et al: Extent and Distribution of Linkage Disequilibrium in Three Genomic Regions. Am J Hum Genet 2001; 68: $191-197$.

30 Abecasis GR, Cookson WO, Cardon LR: The power to detect linkage disequilibrium with quantitative traits in selected samples. Am J Hum Genet 2001; 68, 1463-1474. 